\title{
Estados Unidos y la guerra por el desarrollo: el control de la natalidad en Chile, 1960-1970
}

\author{
Javier CASTRo Arcos \\ Universidad de los Andes, Santiago de Chile \\ jacastro1@miuandes.cl
}

Recepción: 17 de diciembre de 2014 / Revisión: 16 de marzo de 2015

Aceptación: 24 de marzo de 2015 / Publicación: Diciembre de 2015

\section{RESUMEN}

Bajo el paradigma de modernización y desarrollo norteamericano -y en un contexto de Guerra Fría-, durante la década de 1960 se adecuaron a la realidad chilena programas de control de natalidad que no sólo buscaron disminuir las altas tasas de mortalidad materno infantil, sino que además respondieron al proyecto de seguridad hemisférica norteamericana de contención a posibles revoluciones populares en países del Tercer Mundo.

Palabras clave: Guerra Fría latinoamericana, Guerra Fría cultural, Chile, Estados Unidos, control de la natalidad, Historia contemporánea de Chile, explosión demográfica, siglo XX.

\section{The United States and the Struggle for Development: Birth Control in Chile, 1960-1970}

\begin{abstract}
Under the American (United States) paradigm of modernization and development -and within the context of the Cold War- birth control programs were adapted to Chilean realities during the 1960's. These programs not only sought to diminish the high rates of infant and maternal mortality, but they also responded to the American project for hemispherical security, designed to contain potential popular revolutions in Third World countries.
\end{abstract}

Keywords: Cold War in Latin America, Cultural Cold War, Chile, United States, Birth Control, Contemporary History of Chile, Population Boom, $20^{\text {th }}$ Century.

Sumario: 1. Introducción. 2. La amenaza de una explosión demográfica. 3. La guerra por el desarrollo: modernización, Alianza para el Progreso y diplomacia ciudadana norteamericana. 4. Proyecto global, respuesta local: la planificación familiar en Chile. 5. Conclusiones. 6. Referencias bibliográficas. 


\section{INTRODUCCIÓN}

Fueron múltiples las maneras de enfrentar la Guerra Fría en todas sus dimensiones ${ }^{1}$; la diplomacia pública norteamericana abordó un aspecto llamado "guerra por el desarrollo o contra la pobreza", en el cual debía preverse el crecimiento poblacional de los países del Tercer Mundo y los posibles focos de descontrol social, revoluciones masivas, hambrunas, delincuencia y un sinfín de descontentos que los movimientos insurgentes de izquierda utilizarían para sumar nuevos países a la "revolución popular global socialista" 2 . A causa de esta "guerra por el desarrollo" que se estaba debatiendo en el mundo, los lineamientos de la diplomacia pública norteamericana generaron puentes de asistencia técnica y gran apoyo financiero para los programas de control de natalidad que se desarrollarían desde mediados de la década de los años sesenta en Chile.

La noción de "guerra por el desarrollo" se gesta en la administración del presidente Lyndon B. Johnson y, de acuerdo con Francis J. Gavin y Mark A. Lawrence, LBJ incluyó en su agenda intereses ambientales mundiales que anteriormente solo eran considerados por ONG's e instituciones de diplomacia ciudadana y que, inevitablemente, "persistían más allá de la guerra fría". LBJ sentenció que en la Comunidad de Naciones, como en la que se vivía, Estados Unidos no debía ignorar los problemas de los vecinos a riesgo de su propia prosperidad. En este contexto, el presidente norteamericano identificó tres "enemigos" o problemas globales a ser exterminados en esta guerra por el desarrollo: la pobreza, las epidemias y la disminución de los recursos naturales. Este último aspecto fue relacionado expresamente por LBJ con la "explosión de la población mundial" y, para acabar con ese peligro, según su discurso del estado de la unión de 1965, se comprometía a buscar nuevas maneras de utilizar la ciencia y los conocimientos ${ }^{3}$.

Matthew Connelly ahonda en el uso del concepto "guerra" por el desarrollo o contra la pobreza, en la que, según el autor, Estados Unidos asumió el liderazgo de una campaña mundial para el control de la población mundial, y en especial de la reproducción de los pobres en los países pobres:

La inclinación de la administración de Johnson para los análisis de planificación y de costo-beneficio de largo alcance creció para reorientar la política de defensa. Los veteranos de guerra de Robert McNamara pasaron a tomar el control del Pentágono y a liderar la que sería la "Guerra contra la pobreza", aplicando su experiencia a este nuevo escenario -la explosión demográfica ${ }^{4}$.

Esta guerra por el desarrollo se hacía transversal a la Guerra Fría, traspasando los umbrales meramente ideológicos y políticos para instalarse inclusive en la fertilidad de las mujeres. A causa de este tópico, la mujer pasaba a estar en el centro del debate público. Los nuevos estudios de la Guerra Fría se han extendido en visibilizar aque-

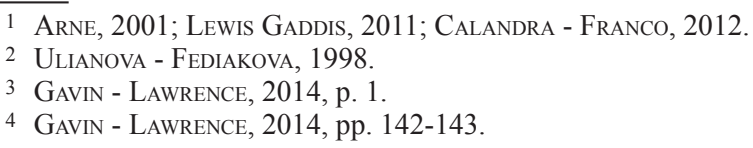


llas aristas soterradas -como el factor demográfico-, propias del clima de la época. De acuerdo con autores como Odd Arne Westad, es conveniente analizar aquellos intentos de Estados Unidos y la Unión Soviética por ir más allá del control del sistema internacional, para influir globalmente en los cambios sociales y económicos, proyectando la imagen de ellos mismos en la vida cotidiana de los individuos ${ }^{5}$.

Los recientes trabajos sobre la Guerra Fría interamericana han ido abriendo nuevas perspectivas para reconocer, dentro de los grandes relatos del clásico bipolarismo, las respuestas locales a desafíos globales, como lo fue la noción de "explosión demográfica" en los países latinoamericanos. En esta línea, novedosos son los trabajos de Joseph Gilbert y Daniela Spencer ${ }^{6}$, Calandra y Franco ${ }^{7}$, Tanya Harmer y Alfredo Riquelme $^{8}$, o el reciente trabajo de Virginia Garrard-Burnet, Mark. A. Lawrence y Julio Moreno' ${ }^{9}$ Todos los anteriores coinciden en mostrar nuevas facetas y temas de investigación en el período, que van desde la diplomacia de la Coca-Cola, o la construcción de imaginarios a través del cine norteamericano, hasta la revolución de la píldora en Brasil entre "bombas y bombones" 10 .

El presente trabajo se propone rescatar un tema no convencional en la clásica mirada historiográfica de la Guerra fría y, por tanto, podría situarse en los nuevos estudios de la Guerra Fría interamericana. Pero a la vez se propone describir y analizar el fenómeno desde una perspectiva institucional en una primera etapa, aunque sin duda son variados las miradas y los actores de esta temática historiográfica que se encuentra en plena discusión.

\section{LA AMENAZA DE UNA EXPLOSIÓN DEMOGRÁFICA}

Al pretender adentrarnos en el reconocimiento del proyecto de modernización norteamericana en Chile, es relevante examinar las implicancias del paradigma neomalthusiano en la política mundial de medidados de siglo. Las antiguas ideas del pastor Robert Malthus, se hacían nuevamente partícipes del debate por el crecimiento de la población, mas ahora en el Tercer Mundo, ya que la "explosión demográfica" en cualquier minuto podía estallar. Socialismo soviético ${ }^{11}$ y Capitalismo norteamericano movían sus piezas de ajedrez para lograr predominio, influencia y disuasión en las dinámicas de poder del globo. Dentro de las variables que se conjugaron entre posibles amenazas mundiales, se halló el factor demográfico y, con mayor detalle,

5 ARNe, 2010. p. 17.

6 Gilbert - Spencer, 2008.

7 Calandra - Franco, 2012.

8 Harmer - Riquelme, 2014.

9 Garrard - LaWrence - Moreno, 2013.

10 Langland, 2013.

11 Este estudio se remite a la influencia estadounidense en las políticas de control de natalidad chilena. Para ahondar en la posición soviética en materias de control de la natalidad, véase SAUVY, 1963; STYCOS, 1968; Consuegra, 1969. 
la "explosión demográfica" ${ }^{12}$ como un elemento que podría presionar y, más tarde, quebrar un deseable equilibrio entre desarrollo económico y paz social.

Las proyecciones de los cientistas sociales y policy makers de la época enseñaron un panorama poco alentador en las regiones del Tercer Mundo ${ }^{13}$. La hecatombe mundial podría avecinarse raudamente y no por el estreno de otra bomba nuclear. La responsabilidad estaría en la falta de medidas concretas para frenar el probable incremento insostenible de la población. El médico chileno Hernán Romero señalaba lo siguiente:

Cada día son menos los que disienten de la opinión prevaleciente de que el crecimiento de población constituye para el mundo en general, y para el subdesarrollo en particular, el problema más grave que ha afrontado la humanidad. John D. Rockefeller ha anotado que supera al peligro de las armas nucleares, puesto que éstas pueden no emplearse jamás, mientras que esa fundación de individuos está constantemente desgastando los recursos y debilitando los cuerpos sociales ${ }^{14}$.

Emergía de esta manera una nueva guerra, la demográfica, que se acompañaba de literatura polémica y sugerente, como el clásico texto de Paul Ehrlich The Population $B o m b$, en el que se manifestaba que la batalla por el alimento debía ser librada ahora, producto de las alarmantes cifras de natalidad.

En Chile, uno de los primeros ponentes del tema fue Armand Mattelart, demógrafo francés que vino a estudiar el control de la natalidad en América Latina. Para él, se había producido en este continente una explosión demográfica, definida como la ruptura del equilibrio secular entre la vida y la muerte. Esta habría engendrado en el lapso de las tres últimas décadas $(1930,1940,1950)$ un desequilibrio de cerca de cien años entre la natalidad y la mortalidad. El impacto de esta supuesta explosión llegó a ser considerada en este período como el segundo problema mundial (connotación que varió según los diferentes países del continente) ${ }^{15}$.

De acuerdo con Karina Felitti, los programas de planificación familiar se contextualizaban bajo un conjunto de intereses que convergían en la materia, en el que se contemplan los esfuerzos de los salubristas por frenar el aborto, las microesferas del poder de decidir la fecundidad por las propias parejas o las implicancias de las demandas feministas -las que aspirarían a una posible revolución del orden de género como parte de un programa de cambio total de la sociedad. Sin embargo, Felitti también reconoce entre estos intereses de fuerte militarización, desconfianza y competencia que imponía el clima de Guerra Fría, la consolidación de un argumento que relacionaba la pobreza de una nación y su falta de oportunidades para alcanzar

\footnotetext{
12 En sencillas palabras, y en línea con la explicación de Poblete Troncoso, los sociólogos y en especial los demógrafos consideran el término "explosión demográfica" como el crecimiento de la población del mundo a un ritmo jamás antes alcanzado que genera gravísimos problemas, o aumenta la intensidad de algunos ya existentes desde antiguo, y que en su conjunto afecta a la supervivencia misma de la humanidad futura. Poblete, 1967. p.15.

13 Stycos, 1968.

14 Romero, 1969, p. 17.

15 Mattelart, 1965, pp. 41-42.
} 
niveles aceptables de desarrollo, con sus altos índices demográficos, reactualizando así una vieja querella generada por las tesis malthusianas a fines del siglo XVIII ${ }^{16}$.

Evidencia del anterior planteamiento, es que el gobierno norteamericano, asesorado por diversas agencias de población, fue construyendo un paradigma que conectaba las variables de seguridad nacional y control natal. El New York Times señalaba entre sus titulares, a fines de los sesenta:

Afirman las autoridades que los Estados Unidos marcharán hacia una catástrofe, a menos que 'controlen' la población del país -y la palabra control está siendo empleada conscientemente en substitución de planificación familiar ${ }^{17}$.

Gradualmente se aprecia cómo la estrategia geopolítica preventiva en materias de desarrollo y tasas de natalidad, se convertía en un nuevo instrumento de influencia filo-norteamericana en América Latina.

De acuerdo con Necochea López, a pesar de que muchos expertos de los Estados Unidos tildaban al crecimiento demográfico en países en vías de desarrollo como una amenaza a su seguridad, no fue sino hasta 1965 que el gobierno de ese país enarboló una serie de reformas a su política exterior para limitar el tamaño de la población más allá de sus fronteras. El NSSM 200 (National Security Study Memorandum) clarifica cómo el Departamento de Estado veía la relación entre la población tercermundista y su propia seguridad. El crecimiento demográfico:

... puede contribuir a incrementar las tasas de abandono infantil, delincuencia juvenil, desempleo y subempleo crónico, crimen, rebeliones, escasez de alimentos, movimientos separatistas, masacres, revoluciones y contrarrevoluciones (NSSM 200, pp.10-11).

Es más, la pobreza y la inestabilidad política alientan la expropiación y la manipulación arbitraria de compañías extranjeras. Ya sea a través de acciones gubernamentales, conflictos laborales, sabotaje o movilización civil, el flujo de materias primas se vería afectado (NSSM 200, p. 37) ${ }^{18}$.

El paradigma de la explosión como asunto de seguridad ya estaba instalado en la alta dirección norteamericana. El Presidente Lyndon Johnson, con ocasión del vigésimo aniversario de las Naciones Unidas, mencionaba en San Francisco:

Afrontemos en todas las tierras, incluyendo esta tierra, los crecientes problemas de nuestras crecientes poblaciones. Busquemos las respuestas a este supremo reto del futuro. Actuemos sobre la base realista de un hecho: 5 dólares invertidos en el control de natalidad equivalen a 100 dólares invertidos en planes de desarrollo económico" ${ }^{19}$.

16 FelitTi, 2008, p. 5.

17 Vaz Da Costa, 1970, p. 219.

18 Necochea López, 2010, p. 104.

19 Bojorge, 1969, pp. 6-7. 
La administración de Johnson declaraba así, explícitamente, la guerra demográfica y hacía pública la declaración de principios rectores de su estrategia en esta guerra por el desarrollo ${ }^{20}$.

Añadiendo a la discusión, el presidente del Banco Mundial, Robert McNamara, situaba su análisis económico precisamente en la brecha de desarrollo entre los países que lograran reducir la natalidad y los que hacían esfuerzos infructuosos por conseguir los objetivos estipulados para acabar con la explosión demográfica. Éste indicaba:

El país que lograra reducir su crecimiento demográfico aumentaría su nivel de vida en un $40 \%$ en relación con el otro país en el curso de una generación. En términos de la diferencia que separa a los países ricos de los pobres estos estudios revelan que, más que cualquier otro factor, la explosión demográfica es la que, al frenar el progreso de los pobres, aumenta la distancia que existe entre los ricos y los pobres y profundiza aun más el peligroso abismo que ya media entre ellos ${ }^{21}$.

Un estudioso del tema, Matthew Connelly, propone en Fatal Misconception: The struggle for Control World Population, la tesis de que este fenómeno global -dentro de un contexto de Guerra Fría- conquistó la agenda local de múltiples regiones del planeta. Para Connelly, de los grandes emprendimientos humanitarios del siglo veinte, no parece haber otro que haya logrado tanto como la campaña de control de la población mundial. Las tasas de fecundidad han disminuido en todas las regiones del mundo y, ahora, las mujeres tienen en promedio la mitad del número de hijos que hace 50 años ${ }^{22}$.

America Latina y Chile fueron regiones de interés hemisferico norteamericano donde los esfuerzos por regular la población respondían a la planificación norteamericana en base a alarmantes estadísticas de proyección global. Por contraparte, la urgencia en la salud pública latinoamericana por frenar altas tasas de mortalidad materno-infantil, requirió la inmediata colaboración norteamericana por medio de la acción de redes científicas y filantrópicas que contribuirían en el modelamiento del paradigma por el Desarrollo que abanderaba la lucha norteamericana en esta región.

\section{LA GUERRA POR EL DESARROLLO: MODERNIZACIÓN, ALIANZA PARA EL PROGRESO Y DIPLOMACIA CIUDADANA NORTEAMERICANA}

A partir del terremoto de Valdivia (1960) -que dejó a Chile en una grave crisis económica- y del impacto de la revolución cubana de 1959 -que constituyó una avanzada

\footnotetext{
20 Es importante recordar que para la administración LBJ, de acuerdo con GAVIN - LAWRENCE, 2014, los "enemigos" de esta guerra por el desarrollo eran: la pobreza, las epidemias, y la disminución de recursos naturales, entre los cuales especialmente éste último aspecto, tenía relación directa con el paradigma de la "explosión demográfica".

21 Discurso de Robert S. Mc Namara, Presidente del grupo del Banco Mundial, ante la Junta de Gobernadores, pronunciado el 30 de septiembre de 1968 en la ciudad de Washington, D. C. El Trimestre Económico, vol. 36, nº 142 (2) (Abril-Junio de 1969), p. 309.

22 Connelly, 2008a, p. 60.
} 
concreta del comunismo en América Latina-, el gobierno del presidente Jorge Alessandri y el del presidente norteamericano John F. Kennedy fortalecieron la mutua cooperación, a fin de prevenir la promoción del comunismo en la región latinoamericana. El proyecto emblemático para sellar el acuerdo anticomunista fue la Alianza para el Progreso, la cual no sólo se realizaría con Chile. Tal como lo enfatizan Castro y Ramos, las circunstancias influyeron en que los Estados Unidos se sintieran presionados por la situación cubana a tender una mano a América Latina en medio de la Guerra Fría. Si bien la Alianza para el Progreso no logró cumplir con todas las metas económicas y expectativas políticas creadas, ha significado la única propuesta, hasta el momento, de los Estados Unidos dirigida hacia América Latina, sin precedentes anteriores y sin semejanzas posteriores ${ }^{23}$.

La Alianza para el Progreso poseía fundamentos conceptuales y teóricos que provinieron en gran parte de la "teoría de la modernización", paradigma predominante en los científicos sociales de la época, incluidos profesores destacados de Harvard y el Massachusetts Institute of Technology (MIT), muchos de los cuales entraron a la administración de Kennedy o se volvieron asesores cercanos. Entre ellos estaba W. W. Rostow, cuyo The Stages of Economic Growth: A Non-Communist Manifesto (Cambridge, Cambridge University Press, 1960) se convirtió en un manual para la Alianza. Participaron también su colega del MIT, Max Millikan y los profesores de Harvard, Lincoln Gordon y John N. Plank, entre otros ${ }^{24}$.

La teoría de la modernización tenía por objetivo ser una propuesta de transformación social y funcionar como ideario que reemplazaría la lucha de clases marxista en pro de una "revolución en libertad", basada en el progreso y el desarrollo socioeconómico, adaptable a la multiplicidad de realidades latinoamericanas a partir de las pautas que de ella se desprendieran. El supuesto teórico poseía tres principales preocupaciones: evolución, progreso y cambio social ${ }^{25}$. Dicha teoría fue sumando audiencia y relevancia en los policy-makers de la administración Kennedy, quienes estaban convencidos de que era posible elaborar y ofrecer una nueva opción estructural para el cambio social en oposición a la revolución puesta en marcha por Castro en Cuba.

Walter W. Rostow, quien comandó el diseño de la modernización, declaró que este consistía en aplicar la teoría económica a la historia económica y entender la interrelación que tienen las fuerzas económicas con otras fuerzas presentes en las sociedades. Con su trabajo, Rostow, quiso dar una explicación alternativa de largo plazo al proceso del desarrollo económico que había dado Marx en el siglo XIX e incluso a la que presentó Joseph Schumpeter, a mediados del XX. ${ }^{26}$ Aún más incisivo, Carlos Quinche, señala que la propuesta Rostow incluía además del objetivo de contener ideológicamente los avances del comunismo por medio del desarrollo económico y la posibilidad de atenuar la pobreza en América Latina:

De acuerdo con la propuesta de Rostow, mediante una importante transfusión de ayuda financiera, los Estados Unidos podrían guiar a casi un $80 \%$ de la población

23 CASTRO - RAMOS, 2014.

24 Lowenthal, 2010, p. 560.

25 MuÑOZ, 1977, p. 78.

26 GutiérReZ, 2004, p. 298. 
latinoamericana por el camino del desarrollo auto-sostenido, lo cual, no solo reduciría la pobreza sino que, al tiempo, lograría disminuir la agitación social y la inestabilidad política en la región ${ }^{27}$.

A la par del significado que tuvo la "teoría de la modernización" en el diseño de la Alianza para el Progreso y en la vinculación paulatina del factor natalidad dentro de las posibles variables de análisis para el desarrollo, existen visiones como la del premio Nobel de economía sueco, Gunnar Myrdal, respecto a la temática. Estas eran bastante consideradas en las recepciones que se hacían en torno a las discusiones que implicaban desarrollo y natalidad. El economista pronunciaba con urgente convicción:

He llegado a la conclusión de que una política exitosa que difunda el control de natalidad supone todo un paquete de reformas que puede dar a la gente la sensación de que viven en una sociedad dinámica que cada vez les abre muchas y mejores oportunidades ${ }^{28}$.

Raúl Prebisch, icono del desarrollismo latinoamericano y precursor de la CEPAL, también se aproximó a la cuestión de la natalidad y, en su criterio, se enfrentaba un fenómeno nunca antes visto en la región latinoamericana:

Quienes estamos confrontados con los problemas del desarrollo económico, estamos poseídos, cada vez más, de una profunda preocupación. La profunda preocupación que los problemas como el crecimiento demográfico y su relación con el desarrollo económico, plantean en estos momentos (...). No se había dado antes el caso de países que pugnan por tecnificar su agricultura y por industrializarse, y -a la vez- tienen una tasa de crecimiento demográfico que rebasa todo lo que históricamente se ha visto antes ${ }^{29}$.

El Cepalismo desarrollista incorporó en sus análisis la dimensión de la planificación familiar en las perspectivas de alcanzar en el mediano plazo un equilibrio demográfico que colaborara con la búsqueda de un progreso autónomo del centro global. Pero, al mismo tiempo, miró con cierto escepticismo aquel birthcontrolismo que veía en el control de la natalidad un fin en sí mismo y casi la panacea para acabar con las carencias de América Latina ${ }^{30}$.

Además del rol que desempeñó la Alianza para el Progreso en la construcción de una voluntad política modernizadora en América latina, también se establecieron profundos vínculos de diplomacia ciudadana por medio de la acción directa en las "bases sociales" chilenas y latinoamericanas. Estas actividades se desplegaron a través de fundaciones, organizaciones y emblemáticos programas como lo fue el Peace $\operatorname{Corps}^{31}$. Estos programas se articularon con los intereses de la política exterior norteamericana -entre ellos la percepción de "explosión demográfica" 32 .

\footnotetext{
27 Quinche, 2009, p. 103.

28 Myrdal, 1974, p. 734.

29 Poblete, 1967. p. 8.

30 PREBISCh, 1992.

31 Cobbs-Hoffman, 1998; Purcell, 2014a.

32 SAUNDERS, 2001.
} 
En cuanto al funcionamiento de dichas organizaciones, fundaciones e instituciones que operan en el mecanismo de la diplomacia ciudadana ${ }^{33}$, Antonio Niño pone de manifiesto que en este período se generó una estrecha colaboración entre la propaganda pública -oficial del Estado norteamericano- y las grandes fundaciones privadas de los años de la Guerra Fría. Ésta se basó en una de las características esenciales del modelo americano de relaciones culturales con el exterior, el principio del laissez-faire y del libre intercambio, sin interferencias gubernamentales, donde las fundaciones cumplían soterradamente los intereses no oficiales de Washington ${ }^{34}$.

De acuerdo con estos planteamientos, Eduardo Rey establece que la actividad de estas fundaciones se enmarca en el concepto de proyección cultural que recogíamos de Niño. Rey destaca la actividad de actores no gubernamentales que, en muchos casos, participan de la labor de difusión de una imagen de país en el exterior y que pueden trabajar a la par de sus gobiernos para alcanzar objetivos compartidos o a cambio de apoyo y/o financiación específica ${ }^{35}$. Esto no significa que estos actores sean siempre instrumentalizados por la política, pues, como ya han mostrado algunos trabajos, comparten una concepción básica sobre el papel internacional que su país y cultura deben jugar en el mundo, lo que los convierte en dos caras de una misma moneda.

En sintonía con Niño y Rey, Fernando Purcell esgrime que en este proceso la gran mayoría de estas instituciones de diplomacia ciudadana consideraron más importante que el anticomunismo discursivo, la idea de acercar la modernidad y el progreso a pequeñas comunidades locales -vistas y consideradas inferiores en sus posibilidades de progreso y, por ende, vulnerables en un contexto de férrea lucha bipolar ${ }^{36}$. Estas entidades llevaron a cabo la vía de guerra "pacífica" y cultural contra la pobreza, a la vez que se conformaron al contener los posibles embates que podría encender el socialismo en vista de las carencias y descontentos populares que florecían en extensas ciudades latinoamericanas.

Bajo la premisa del progreso y desarrollo capitalista en todo el continente, fundaciones y organizaciones transnacionales como la Rockefeller, Ford e IPPF ${ }^{37}$ tomaron la bandera de la planificación familiar como asunto de alta prioridad en la implementación de los modelos de desarrollo para el Tercer Mundo latinoamericano. No obstante, ¿a qué más se debía la preocupación norteamericana en materia de control de la población hacia el Tercer Mundo?

Como hemos dicho desde un principio, la planificación familiar involucró esfuerzos voluntarios de actores no gubernamentales, pero -y en sintonía con los planteamientos de Connelly- ese enfoque pareció cada vez más insuficiente cuando comenzó la Guerra Fría y pasó a ser un argumento de seguridad nacional de primer plano para los Estados Unidos. “... No estamos interesados principalmente en los aspectos sociológicos o humanitarios de control de la natalidad”, escribió Hugh Moore, el cofundador del Comité Norteamericano de Crisis de Población. "Estamos interesados

33 Cull, 2008; Hart, 2012.

34 NiÑo, 2009, p. 41.

35 Rey Tristán, 2012, p. 59.

36 Purcell, 2014b.

37 Calandra, 2011; Cueto, 1994; Necochea, 2014. 
en el uso que los comunistas quieren hacer de las personas que padecen hambre en su afán de conquistar la tierra" ${ }^{38}$. En sencillas palabras, el control de natalidad, dentro del contexto de Guerra Fría, puede ser considerado como una estrategia de contención anticomunista en el Tercer Mundo.

En términos generales y en relación con lo que asevera Michael Latham, observamos que las superpotencias asumían que su seguridad nacional dependía de la difusión de sus visiones de modernización o transformación socialista -dependiendo del caso. Esta proyección los llevó a desplegar una intensa fuerza de persuasión en su propagación. En definitiva, muchas élites del Tercer Mundo, ávidas de un rápido progreso económico y social, también abrazaron aquellos enfoques, llegando algunos a emplear, inclusive, la represión en nombre de la transformación ${ }^{39} \mathrm{y}$, otros, a adecuar paquetes de decididas reformas, como lo fue la planificación familiar, en pos de alcanzar los estándares que se desprendían de la propia teoría de la modernización.

Según Latham, proyectos como la Reforma Agraria y el control de la natalidad daban cuenta de intervenciones prescritas que dependían, en gran medida, de la correcta aplicación de estos métodos de ingeniería social, los que buscaban transformar campesinos tradicionales en ciudadanos modernos capaces de producir -supuestamentemás recursos, a la vez que deseaban tener menos hijos ${ }^{40}$. La opción del salto hacia el desarrollo estaba estipulada metodológicamente en las fórmulas estructuralistas que se habían planteado, bastaba la voluntad política y el arrojo para aceptar el desafío de nivelar a los supuestos señalados el curso de la historia.

En Chile, el gobierno demócrata cristiano de Eduardo Frei Montalva ejecutaría en gran medida parte del programa modernizador que se desprendía de las agencias de planificación norteamericana ${ }^{41}$. La consigna de su campaña presidencial, Revolución en Libertad, dejaba interpretar estos supuestos, donde se promovía, como eje central, el desarrollo para todos los chilenos.

\section{PROYECTO GLOBAL, REPUESTA LOCAL: LA PLANIFICACIÓN FAMILIAR EN CHILE}

El intelectual e historiador chileno Mario Góngora denominó el período de los años sesenta como la era de las grandes planificaciones, ya que, según él, el espíritu del tiempo tendía en todo el mundo a proponer utopías (o sea, grandes planificaciones) y a modelar conforme a ellas el futuro. La idea era partir de cero, sin hacerse cargo ni de la idiosincrasia de los pueblos ni de sus tradiciones nacionales o universales; la noción misma de tradición parecía abolida por la utopía. En el caso de Chile, la empresa parecía tanto más fácil, pues su resorte último era "técnico-económico-masivo, no un alma" ${ }^{42}$. Vemos que en la era de las planificaciones, específicamente la década de los sesenta, es también símbolo de revoluciones y reformas mundiales, de carac-

\footnotetext{
38 Connelly, 2008b, p. 61

39 LATHAM, 2011, p. 280.

40 Latham, 2010, p. 94.

41 Korry, 1998.

42 GóngOrA, 1981, p. 138.
} 
terísticas políticas, sectoriales, agrarias y, además, fue la era de la llamada revolución de la píldora.

En el caso chileno es importante mencionar que la década de los sesenta también tuvo un carácter movedizo y revolucionario, de cambios que se respiraban en todos los ámbitos y sectores de la sociedad. Sofía Correa caracteriza e interpreta este decenio como una "bisagra histórica", en la que particularmente los jóvenes y las mujeres ascendieron como figuras que encarnaban las transformaciones y proclamas del período:

La efervescencia social, la transgresión a las costumbres, el desenfreno eufórico por el cambio y un fuerte optimismo y confianza en el futuro, fueron los signos que marcaron la pauta. Fue una época de trastornos en las modas, estéticas, consignas, representaciones y conductas, liderada por sujetos nuevos como los jóvenes y las mujeres en el nuevo marco de una cultura de masas que se consolidaba, todo lo cual irrumpió en la vida pública con inusitada magnitud. La década se convirtió en una verdadera bisagra histórica ${ }^{43}$.

Sin duda -y como indica Joaquín Fermandois-, los años sesenta en Chile presenciaron una creciente ebullición. En este período la polarización política se incrementó notablemente. La insatisfacción con el "sistema" era patente, lo cual, según Fermandois, creó un campo de fuerzas que parecía temible o excitante, pero de difícil manipulación y, a veces, contradictorias entre las propuestas del bipolarismo internacional en la escena local ${ }^{44}$.

Por su parte, el Cepalismo chileno en la era de las planificaciones globales consideró críticamente la modernización de Rostow (cuestionamientos claros en la "Teoría de la Dependencia" cepaliana). Sin embargo, en materias poblacionales ambas escuelas de interpretación económica (modernizadora y cepaliana) eran planificadoras y consideraban el factor población como un elemento clave a la hora de proyectar un desarrollo socioeconómico. Los planificadores como el economista Jorge Ahumada, al referirse al crecimiento demográfico, argumentaban ineludiblemente:

Si hay cada vez más personas, pero la misma cantidad de bienes, los bienes disponibles por persona disminuirían inevitablemente, y en definitiva cuando la producción crecía a un ritmo inferior al del crecimiento demográfico, las dificultades sociales que surgirían de la frustración de los que no encuentran un modo digno de ganarse la vida, serían al menos conflictivas para el bienestar social general del país ${ }^{45}$.

Los intelectuales del cepalismo no callaron la posibilidad de hacer valedera una ecuación para el desarrollo que incluyera el factor población. Un tanto más acucioso, Aníbal Pinto Santa Cruz, escritor del clásico Chile, un caso de desarrollo frustrado, reflexionaría en torno a la tendencia que significaba la explosión demográfica en Chile y América Latina. Lo interesante es que Pinto, además de replicar a favor de la

43 CORREA, 2001, p. 226.

44 FermandoIs, 1998, p. 157.

45 Ahumada, 1958, p. 57. 
planificación en términos económicos, realiza una crítica socioeconómica de fondo ante el asunto del control de natalidad, llegando a aseverar que debía ser calificada más bien como la "explosión de los pobres":

La trascendencia de la explosión demográfica, en sí misma y en relación al problema del desarrollo, va acrecentándose a medida que se alargan las perspectivas. Y nadie dejaría de inquietarse profundamente ante las proyecciones de la población y el análisis de sus implicaciones (...). Desde luego, los grupos de ingresos medios y altos han conseguido por diversos medios, más o menos "civilizados", reducir el tamaño y el crecimiento de sus núcleos familiares y en parte lo mismo sucede con las clases populares, aunque en este caso los procedimientos son, por lo general, bárbaros -como lo acreditan los pavorosos antecedentes sobre el aborto en la región-, y además, ineficaces, como lo demuestran las tasas de crecimiento poblacional. Vale la pena recordar que la llamada "explosión demográfica" es en realidad la "explosión de los pobres" ${ }^{4}$.

Los asuntos poblacionales son conectados directamente con los indicadores de $D e$ sarrollo que pensaban los economistas y analistas de la CEPAL, generarían un punto de equilibrio para un desarrollo igualitario. Las series de estudio de CELADE y las notas de Población de la CEPAL dan cuenta de esta realidad ${ }^{47}$.

Así como los cientistas sociales, múltiples médicos manifestaron su inquietud en relación a la existencia de programas científicos enfocados a la planificación familiar en Chile, apuntando a buscar soluciones urgentes a las altas tasas de mortalidad infantil, presentes como una endógena problemática chilena ${ }^{48}$. De acuerdo con el Dr. Onofre Avendaño, los primeros apoyos económicos internacionales para esos programas comenzaron a llegar en 1963 a través de la Federación Internacional de Planificación de la Familia (IPPF), Región del Hemisferio Occidental. Asimismo, durante aquel mismo año, organismos privados de carácter internacional como el Consejo de Población (Population Council), la Fundación Ford y otros se interesaron en promover en el país actividades de investigación en reproducción humana y de la regulación de la fertilidad ${ }^{49}$. Sin embargo, existen antecedentes que muestran que ya desde febrero de 1954 el Servicio Nacional de Salud chileno había recibido fuertes sumas de dinero a través de la Rockefeller y Ford Foundation, dinero que fue usado para que Naciones Unidas instalara dos centros de investigación demográfica mundial, de los cuales uno se instaló en India y el segundo en Chile ${ }^{50}$.

Se sumaron a este propósito, en el sentido de financiar campañas que propendieran hacia el control de los nacimientos, la ONU, el Banco Mundial e importantes agencias de financiamiento internacional, tales como la Agencia para el Desarrollo

\footnotetext{
46 PINTO, 1968. pp. 57-58.

47 Véase: Repositorio digital de la CEPAL/CELADE. En: http://repositorio.cepal.org

48 Según Gonzalo Vial, "Tocante a los niños los guarismos con toda frivolidad son intolerables. Sobre 626.623 fallecidos desde 1905 hasta 1910, 303.417 (48,42\%) no alcanzaban los cinco años. ¡Durante aquel sexenio, día tras día, hora tras hora, murió en Chile un niño cada menos de diez minutos! Sobre 100 nacidos vivos, antes del año perecían 30,34 incluso 38 (1917). Compárese tal cantidad con las de Londres $(11,4)$, Buenos Aires $(10,5)$ y la insalubre Río de Janeiro $(12,3)$ ”. VIAL, 1981, p. 537.

49 Avendaño, 1975, pp. 10-11.

50 Connelly, 2008b, p. 159.
} 
Internacional (AID). Ahora bien, el catalizador para la implementación del control de natalidad en Chile fue el programa de la Alianza para el Progreso ${ }^{51}$, sobre todo por los canales de interrelación oficial que esta ofrecía para el financiamiento de las organizaciones internacionales. Claudia Rojas expone la forma en que se articuló el proyecto de planificación familiar desde la Alianza para el Progreso:

En 1965, una Oficina de Población fue formalmente establecida como parte de la Alianza Para el Progreso y fue considerada como la principal innovación en materia de asistencia extranjera. Teodoro Moscoso, director de la Alianza, había estado involucrado profundamente en la planificación de la población de Puerto Rico en los años cincuenta. Moscoso nombró a Edgard Berman para encabezar la nueva de la Alianza, había estado involucrado profundamente en la planificación de la población Oficina de Población y desarrollar estrategias con el propósito de convencer a otros gobiernos latinoamericanos para adoptar políticas de control de la población. La experiencia de Moscoso y el éxito del experimento en Puerto Rico, pudo persuadir a la Comisión de Relaciones Exteriores del Senado de los Estados Unidos y así sancionar la primera subvención del Departamento de Estado en Latino América, dirigida a la Organización Panamericana de Salud (OPS) y al CELADE, centro de investigación demográfica patrocinado por las Naciones Unidas con sede en Chile, con fondos de las Fundaciones Rockefeller y Ford, así como la Agencia Para el Desarrollo Internacional (AID). El CELADE se había establecido en 1957, mediante un acuerdo entre Chile y las Naciones Unidas cuyo objetivo principal era enseñar demografía y promover actividades en ese campo ${ }^{52}$.

Como también lo estipulan Levinson y De Onis, con la influencia de la Alianza para el Progreso, el control de la natalidad y población llegó a ser un tema relevante en la agenda política regional. Por ejemplo, en 1966 en una reunión de la OEA, se propuso discutir la relación entre política demográfica y desarrollo y, en 1968, Estados Unidos destinó 25 millones de dólares al programa de ayuda para América Latina, con el fin de financiar proyectos de planificación familiar, de investigación científica sobre reproducción y estudios de población ${ }^{53}$. Quintana confirma lo determinante que fue la Alianza, ya que durante los diez años en los que esta se ejecutó, la difusión de la píldora anticonceptiva fue, quizás, uno de los resultados con más fuerte impacto social en la región. A pesar de las oposiciones, para 1970 el número de usuarias de la píldora se calculaba en 2,5 millones, la mayoría entre clases medias y altas ${ }^{54}$.

Las acciones programáticas y de prestación de servicio materno infantil inmediatas fueron ejecutadas por la Asociación Chilena de Protección a la Familia (APROFA),

51 "Since 1965 the United States has contributed more to foreign population control programs tan all other countries combined and has pressured other countries and international agencies to back the program. In addition to more than 2 billion dollars in explicit AID 'population assistance' appropriations to various countries and international organizations - including the World Health Organization, the Food and Agriculture Organization, UNESCO, UNICEF, and the International Labor Organization -that have been used for population control, with a degree of enthusiasm and dedication equal to that of the AID bureaucracy". En KASUN, 1988, p.79.

52 RoJas, 2009, p. 9.

53 LeVInSON - De OnIS, 1972. p. 42.

54 Quintana, 2010, pp. 42-43. 
que ofició de organismo central en la masificación de la píldora y en la dirección logística y técnica de los programas de planificación familiar en Chile. De acuerdo a Heidi Tinsman, los representantes de APROFA actuaron como consejeros en el programa de Promoción Popular que desarrollaría el gobierno demócrata cristiano del presidente Eduardo Frei Montalva (1964-1970), en la coordinación nacional de los Centros de Madres y en el Departamento de Planificación Familiar del SNS. Tinsman llega a detallar que, durante la administración Frei, el costo de los programas de cuidado materno y de control de la natalidad fue asumido por el gobierno de los Estados Unidos y por fundaciones privadas estadounidenses. Entre 1964 y 1970, Chile recibió más de US\$ 5.000.000 para implementar iniciativas de planificación familiar, los que provinieron de contribuciones combinadas de USAID, International Planned Parenthood, The Pathfinder Fund, The Ford Foundation y, especialmente, de la Rockefeller Foundation. Según estimaciones de APROFA, el 50\% del financiamiento de la difusión de los métodos de control de natalidad durante el gobierno de Frei estuvo en manos de la Fundación Rockefeller ${ }^{55}$.

El interés de los de Estados Unidos estaba en fortalecer y auspiciar el desarrollo de Chile con el propósito de que se convirtiera en un país lo suficientemente estable y confiable como para servir de modelo regional e ícono de reformas por vías anticomunistas, explica que también valiera la pena una inversión económica y social estadounidense $^{56}$. Por lo tanto, todas las opciones de fortalecer el paradigma modernizador hacia el desarrollo -vinculado al aspecto natalidad-, serían notoriamente potenciadas. De esta forma, además del influjo operativo de APROFA, se desarrolló un pensamiento chileno contronatalista asociado al desarrollo económico.

El destacado médico chileno Benjamín Viel -quién más tarde sería un agente global de la IPPF de New York- desarrolló en la década una batería teórica que nutrió a Chile y América Latina de un pensamiento neomalthusiano en materias de reproducción sexual y regulación de la natalidad. En su destacado escrito La explosión demográfica, daba a conocer la urgencia regional por la revolución demográfica:

Frente a una América Latina que sufre los efectos de una revolución demográfica antes de obtener los beneficios de la revolución industrial, el demorar una política de población significa favorecer el círculo en el cual el aumento engendra miseria y dolor y arrastra a una catástrofe que puede destruir muchos valores que el hombre debe tratar de conservar ${ }^{57}$.

Un tanto más escéptico de lo que denominaba "birthcontrolismo" e informado de las dinámicas y esfuerzos globales de esta guerra por el desarrollo, Radomiro Tomic Romero, el embajador de Chile en Estados Unidos durante el gobierno de Eduardo Frei Montalva, se mostraba firmemente convencido de que la única manera de alcanzar el desarrollo en América Latina y Chile era por medio de la modernización de las instituciones. No obstante, asumía la necesidad de la planificación familiar en casos de miseria socioeconómica. Entre sus cuestionamientos planteaba la factibilidad del

\footnotetext{
55 Tinsman, 2009, p. 171.

56 KORRY, 1998.

57 VIEL, 1966, pp. 232-233.
} 
uso de la presión demográfica en torno a las variables: uso del territorio, modernización de las instituciones y control de la natalidad.

¿Se ha reflexionado acaso cuidadosamente en el hecho de que probablemente el factor aislado más importante para dar impulso al progreso de modernización de las sociedades o países subdesarrollados, es precisamente la presión social creada por el crecimiento de la población? ${ }^{58}$.

La cuestión del control de la natalidad no quedaba tampoco exenta de debates en las perspectivas religiosas y, en específico, de la Iglesia Católica. De acuerdo a Correa, el Cardenal Silva Henríquez y otras congregaciones religiosas sintonizaron con las demandas de la época y con la Alianza para el Progreso ${ }^{59}$. Interesante resulta ver la opinión de Silva Henríquez respecto a las promesas de ese programa, ya que en los mismos años se publicaba la encíclica Humanae Vitae ${ }^{60}$, que condenaba directamente la regulación de la natalidad. Como lo indica Bojorge, Humanae Vitae es la piedra de tropiezo que hace estallar una extraña e inesperada dicotomía entre los dos hemisferios en la más moderna e insidiosa de las guerras: la demográfica. Una guerra -según Bojorge- que, a pesar de la metamorfosis de su rostro, es tan mortal, tan inmoral y tan injusta como cualquier otra ${ }^{61}$. Silva Henríquez y Frei tuvieron que enfrentar el dilema y, de acuerdo a una entrevista llevada a cabo al Doctor Ramiro Molina ${ }^{62}$, las negociaciones habrían sido de la siguiente manera:

Los doctores Jorge Rosselot y Francisco Mardones (Director del Servicio Nacional de Salud (1952) llevan al Presidente Frei el registro de los números y tendencias de mortalidad materna por causa de los abortos. Frei reacciona con la urgencia de prevenir el asunto, con un programa de regulación de la fecundidad. Frei les dice está bien pero primero me traen el acuerdo del cardenal Silva Henríquez. La comitiva de los médicos en su gran mayoría eran masones. Sin embargo la negociación se llevó a cabo, tras una "tacita de té" que duró desde las cinco de la tarde hasta las tres y media de la mañana, las tratativas se concretaron. Impresionante fue para la comitiva cuando el Cardenal Silva Henríquez les declaró, 'Ustedes los laicos tienen que aprender a asumir la responsabilidad que les corresponde... No vengan a pedir permiso a la Iglesia Católica, no se vengan a esconder en la sotana de los curas para lo que ustedes tienen que hacer, aprendan a asumir sus responsabilidades, pero nosotros nos guardamos el derecho de velar por el derecho de los niños, de la mujer entre otros...'. La comisión se fue al Ministerio de Salud, comunicaron a primera hora a Frei la conversación y a las ocho de la mañana el decreto estaba aprobado y listo para todo Chile" (agosto de 1965) ${ }^{63}$.

58 Tomic, 1966, p. 21.

59 Correa, 2001, p. 216.

60 "El problema de la natalidad, como cualquier otro referente a la vida humana, hay que considerarlo, por encima de las perspectivas parciales de orden biológico o psicológico, demográfico o sociológico, a la luz de una visión integral del hombre y de su vocación, no sólo natural y terrena sino también sobrenatural y eterna". PAUlo VI, 1968, cap. VII.

61 Bojorge, 1969 , p. 7.

62 Fue Directivo de APROFA en la década del `60, Master en Salud Pública por la John Hopkins University, que fue parte de los programas de becas y especialización de demógrafos para Chile.

63 Entrevista al Dr. Ramiro Molina, 27 de agosto de 2012. Santiago de Chile. Ver además Lagos, 2001, p. 86 . 
Las disputas y cuestionamientos siguieron abiertos. Muchos se opusieron a la tratativa que expandió y oficializó el plan de promoción popular de regulación de la natalidad de los demócratas cristianos. Sin embargo, dentro de la misma Iglesia, existieron opiniones controvertidas que terminaron por consolidar el control de la natalidad en la población chilena. Conviene destacar, por relevantes, las opiniones del sacerdote jesuita Hernán Larraín en la revista Mensaje de enero de 1966:

... No se trata aquí primariamente de promover el uso de anticonceptivos (¡no abortivos!) en cuanto tales sino de permitirlos para impedir males mayores: aborto, irresponsabilidad frente a los hijos, daños físicos, psicológicos y morales. En cuanto a la campaña que propicia el SNS, va dirigida a defender la salud de la madre y del hijo, y el bienestar de la familia; es algo no solo lícito, sino encomiable y, por supuesto, puede y debe el médico católico colaborar en ella. Del momento que este objetivo solo se puede lograr permitiendo el uso de anticonceptivos, puede el médico católico hacerlo en función de los principios ya indicados para evitar un mal mayor ${ }^{64}$.

Continuaron los debates valóricos, económicos y culturales a nivel local. No obstante, el control de la natalidad era legitimado internacionalmente a través de la VIII Conferencia Internacional de la International Planned Parenthood Federation en Chile, la semana del 8 al 16 de abril de 1967. La IPPF llevaba a cabo su primera conferencia internacional en América Latina. El lugar seleccionado había sido Chile, país que fue aceptado como miembro titular de la Federación Internacional de Planificación de la Familia, el 17 de septiembre de 1965 en reunión del Cuerpo Gobernante de la Federación, celebrada en Londres. Esta Conferencia situaba al país del extremo sur en la vanguardia del movimiento mundial contronatalista. Durante estos días se trabajaría para que el trabajo de la IPPF y de las diversas organizaciones asociadas a ella, cumplieran con los siguientes objetivos:

$1^{\circ}$ Influir en un mayor número de gobiernos, en un mayor número de países.

$2^{\circ}$ Asegurar que la regulación de la natalidad no permanezca como entidad aislada sino que, por el contrario, se integre en los servicios de salud y bienestar, procurando que los dirigentes de la comunidad, los economistas, los industriales, los jefes sindicales, los maestros y todos aquellos individuos interesados en el desarrollo reconozcan el valor de esta integración.

$3^{\circ}$ Influir en las universidades y en las escuelas de medicina para que se incorpore la enseñanza de técnicas anticonceptivas en el plan de estudios de dichas instituciones.

$4^{\circ}$ Estimular el interés y la cooperación de la profesión médica y del personal paramédico, a fin de que promuevan la práctica de regulación de la natalidad dentro de sus propias comunidades, durante el desempeño de sus labores habituales.

$5^{\circ}$ Incrementar los servicios que actualmente ofrecen las asociaciones miembros ${ }^{65}$.

64 LARRAín, 1966, p. 46.

65 Boletín nº 4. Año II APROFA Asociación Chilena de Protección de la Familia. Abril de 1966. p. 2. 
Destacable fue además la colaboración de 1.300 .000 de escudos $\left(\mathrm{E}^{\circ}\right)$ de parte de la Agencia Internacional para el Desarrollo de Estados Unidos (AID) ${ }^{66}$ durante el año 1967, donde gran parte de estos fondos no solo auxiliaron parte de lo que significó la Conferencia, sino que también era destinado para la ayuda y asistencia del sistema de salud pública chilena. El presidente de la República, Eduardo Frei Montalva, se encargó de abrir la ceremonia y, en su discurso inaugural, declaró:

Tenemos la mente muy abierta para considerar el problema. No tenemos frente a él ningún prejuicio, solo queremos proceder con la mayor seriedad posible, porque, si bien es cierto que, en general, la política es signo muchas veces de enjuiciamiento, yo no puedo olvidar que Aristóteles la colocó como la primera de las ciencias. En último término los hombres expertos elaboran las soluciones, pero los gobiernos deben tomar la decisión y la responsabilidad de aplicarlas con el consentimiento de su propia nación. Por esta razón he recogido con extremo interés las palabras de Lord Caradon. El nos ha dicho que no solo es necesario considerar y respetar los derechos humanos, sino que éste es un problema que debe resolver cada familia y cada nación, por las particulares condiciones y problemas que a cada una presenta. También estamos muy conscientes de que, en esta época, el problema de la explosión demográfica no se puede eludir, que no basta estudiarlo sino que hay que resolverlo, afrontándolo con valor. Es un problema que no solo afecta a cada familia y a cada estado sino a la humanidad entera $^{67}$.

La conferencia cumplía el objetivo de perfilar a Chile en la vanguardia de materias de planificación familiar y, desde entonces, se comenzó a distinguir ese país como modelo para el desarrollo en América Latina.

Durante la misma semana se llevó a cabo la Conferencia de Presidentes latinoamericanos en Punta del Este. Sin vacilación, APROFA hacía el nexo entre la conferencia de la IPPF en Santiago y la de mandatarios en Punta del Este, bautizando esta semana como una de las más importantes de la historia americana:

... Esta semana ha sido especialmente trascendente en la historia del mundo, y en especial de América. Al mismo tiempo que 87 países estaban representados en esta reunión, dieciocho gobernantes de América asumían en Punta del Este el compromiso de adoptar aquellas medidas que permitieran, dentro del más breve plazo, garantizar el Desarrollo de América Latina ${ }^{68}$.

En una comparación de Conferencias, The New York Times, con fecha del 11 de abril de 1967, hacía énfasis en señalar lo siguiente:

\footnotetext{
66 AMRE, vol. I, $\mathrm{n}^{\circ}$ 182. Ministerio de Salud. Oficios reservados y ordinarios intercambiados con el Ministerio de RR.EE. de Chile 1967. Asesoría Técnica Programas Internacionales. Oficio Nº80. “Acciones frente a la AID". 23 de mayo de 1967.

67 Federación Internacional de Planificación de la Familia. Actas de la Octava Conferencia Internacional de la Federación Internacional de Planificación de la Familia, Santiago de Chile, 9-15 de abril de 1967. Paternidad consciente, deber y derechos humanos. Hertfordshire. Stephen Austin and Sons, 1967. p.16.

68 Boletín $\mathrm{n}^{\circ}$ 5. Año III APROFA Asociación Chilena de Protección de la Familia. Santiago de Chile. Mayo de 1967, p. 7.
} 
Ninguna región del mundo tiene mayor necesidad de controlar su población que América Latina. La Conferencia de Santiago podría tener mayores efectos potenciales que la reunión de Presidentes que comienza mañana (12 de abril) en Punta del Este. La conferencia de Chile es verdaderamente de carácter internacional ${ }^{69}$.

La importancia que asignaba el New York Times a la Conferencia de la IPPF en Chile, probablemente se circunscribía al fuerte despliegue mediático que tenía el paradigma de la explosión demográfica en recientes discursos como los de LBJ en Naciones Unidas o por literatura y reportajes que ponían el tema permanentemente en tela de juicio. Por otro lado es interesante la jerarquización de relevancia que hace el periódico, priorizando una problemática global, antes que una regional.

Durante los mismos días de aquella semana de abril coincidieron en Santiago citas regionales altamente relevantes, que pudieron haberse coordinado de manera deliberada o pudieron ser fruto del mero azar. Al tiempo que se efectuaba la VIII conferencia de la IPPF en Santiago, se iniciaba también la segunda reunión del Comité de Planificación del Desarrollo de las Naciones Unidas. El diario La Nación aseguraba que esta reunión contaría con más de 18 especialistas mundiales:

Los expertos analizarán las metodologías de las proyecciones económicas mundiales y los preparativos para una acción internacional en el periodo que siga al actual decenio de las Naciones Unidas para el Desarrollo. La ceremonia inaugural será presidida por el Subsecretario de Asuntos Económicos y Sociales de la Organización de las Naciones Unidas, Phillipe de Saymen. Por otra parte, la Comisión Económica para América Latina, CEPAL, ha preparado un documento que servirá de base a las deliberaciones de esta conferencia. Este informe señala que en la presente década, Chile, Colombia y Bolivia fueron los primeros países latinoamericanos que decidieron emprender la formulación de planes nacionales de desarrollo, y empezaron a establecer los mecanismos correspondientes, apoyados en buena medida por el asesoramiento técnico internacional ${ }^{70}$.

Simultáneamente la Comisión Interamericana de Mujeres (la CIM era una organización de la OEA que, en aquel período, representaba a más de 100 millones de mujeres) había convocado, aquella misma semana, a sus representantes de Argentina, Bolivia, Brasil, Chile, Colombia, Ecuador, Panamá, Paraguay, Perú, Uruguay y Venezuela en el segundo curso de adiestramiento para mujeres dirigentes, que lideró su presidenta, la costarricense Margarita de Macaya. Chile colaboró en este curso por medio de la participación de la Universidad de Chile, la Consejería Nacional de Promoción Popular, la Universidad Católica y el Comité chileno de Cooperación a la Comisión Interamericana de Mujeres. En su realización la CIM recibió el apoyo financiero del Consejo Interamericano Económico y Social (CIES), del Banco Intera-

69 Boletín n ${ }^{\circ}$ 6. Año III APROFA Asociación Chilena de Protección de la Familia. Santiago de Chile. Junio de 1967 , pp. 2-3.

70 La Nación. "Segunda reunión del Comité de Planificación de Desarrollo de Naciones Unidas". 10 de abril de 1967, p. 17. 
mericano de Desarrollo (BID) y del Comité Panamericano de Organizaciones femeninas $^{71}$. Margarita de Macaya, recalcó a la prensa nacional el objetivo de la reunión:

La mujer quiere actuar en forma positiva para lograr que la humanidad sacie su hambre fisiológica y su hambre espiritual. Quiere capacitarse para esta lucha en todos los niveles -continuaba La Nación. ... Margarita de Macaya, una vez instalado el curso, viaja hoy a Punta del Este, Uruguay, para estar presente en la Conferencia de Presidentes, en cuya declaración final la CIM obtuvo la inclusión de la mujer como una de las grandes protagonistas de la hora actual para toda América ${ }^{72}$.

La interrelación de conferencias regionales en la segunda semana de abril de 1967 en Santiago de Chile -y también en Punta del Este- se ajustó al propósito del desarrollo como bandera principal en un plan global de masificación que incluyó el contronatalismo, siempre abordado desde las perspectivas económicas, sociales, feministas y de seguridad hemisférica, entendida dentro de la dinámica propia de Guerra Fría en el Tercer Mundo.

La organización de las Naciones Unidas, por su parte, promovió la búsqueda de una coordinación estratégica global para la masificación de programas de planificación familiar y lo hizo notar en la Conferencia de la IPPF, por medio del destacado diplomático chileno Hernán Santa Cruz. Este, en su calidad de Director General Adjunto de la FAO para América Latina, manifestó su fe en que la Humanidad ganaría la batalla por la supervivencia, pero a condición de que se aplicara una estrategia global contra el subdesarrollo:

Insistimos -dijo Santa Cruz- en que ambos frentes, el de la regulación de la familia, y el de la mayor producción, constituyen la respuesta al desafío. El instrumento de la victoria es el desarrollo económico y social. ... Hoy día que la ciencia y la técnica, con sus avances prodigiosos, han multiplicado el número de habitantes, reducido las distancias e independizado a todas las naciones del globo en un grado superlativo, aquellos problemas solo tendrán solución si la humanidad entera conjuga sus esfuerzos y sus recursos con sentido solidario ${ }^{73}$.

Santa Cruz difundió el discurso global desarrollista, la agenda en la que también Naciones Unidas se hallaba trabajando bajo similares parámetros y lo promovió en la opinión pública chilena libremente. La lectura que se hacía es que aquel era el momento de América Latina y que, por tanto, Chile podría oficiar de satélite regional para las políticas planeadas globalmente. La opinión de la historiadora norteamericana Jadiwga Pieper es que por medio de esta instancia se generó un compromiso en donde diversos funcionarios del gobierno chileno continuarían asumiendo la responsabilidad de desarrollar programas nacionales en consonancia con las iniciativas globales dirigidas al desafío que ofrecía el crecimiento de la población. Pieper indica que, a pesar de que Frei no se refirió a los nuevos "derechos" de las mujeres locales,

71 La Nación. "La mujer hacia un futuro mejor". Martes 11 de abril de 1967, pp. 1 y 6.

72 Ibídem.

73 La Nación. "Menos hijos o más pan." Martes 11 de abril de 1967, p. 5. 
de las madres chilenas, otros dejaron en claro que se esperaba que Chile desempeñara un rol crucial en iniciativas de este tipo ${ }^{74}$.

\section{CONCLUSIONES}

El aterrizaje en Chile de la dimensión global que comprendió el movimiento de planificación familiar, se situó con efectividad específicamente en los años en que Estados Unidos promovió e instaló su modelo de modernización y desarrollo regional a través de la Alianza para el Progreso. La constante crisis de mortalidad materno-infantil y las altísimas tasas de aborto que había arrastrado Chile en su historial de salubridad pública, fueron transformadas en la década de los sesenta, precisamente, en la etapa de mayor influencia económica, política e intelectual del gobierno norteamericano $\mathrm{y}$, asimismo, al tiempo que las organizaciones filantrópicas ligadas a él realizaron esfuerzos claros por modelar la realidad socioeconómica de la familia chilena.

La cuestión de la natalidad había sido resuelta y los indicadores ofrecerían cifras alentadoras en los próximos años. Por ello, según los doctores que fueron conductores de la implementación de los programas, Francisco Mardones y Guildo Zambra, el Departamento de Estado Norteamericano señaló a ese país como cabeza de los programas de control de la natalidad en América Latina. Chile sirvió como base de operaciones para el desarrollo de proyectos de regulación de la fecundidad en la región entera, casi como un "aleccionador" 75 . La década de los sesenta fue un período en donde los médicos chilenos volcaron sus esfuerzos para frenar la epidemia de abortos y regular la población. La especialización y la red de colaboración científica que se estableció en el transcurso de la década, potenció a los médicos chilenos como ejemplo en la ejecución de programas de planificación familiar. Cada año se graduaban cerca de treinta médicos expertos en población y transición demográfica ${ }^{76}$. Chile funcionaba como modelo regional exitoso en materias de control de la natalidad y regulación de la explosión demográfica.

Posterior a la Conferencia de la IPPF, el proyecto de desarrollo se incorporó y se vio materializado determinantemente en la institución de la Salud Pública chilena, por vía de la implementación de programas de control de la natalidad. Eso trajo consecuencias de alto impacto en la amplia gama de políticas públicas del Estado, determinando el transcurso demográfico de las próximas generaciones chilenas. Influyó en una nueva actitud de la mujer, y, por lo tanto, en la construcción de una sociedad distinta, subjetiva y emancipada de un concepto tradicional de familia, heredado de la antigua matriz cultural de raigambre hispana.

Tal como lo indica el Dr. Enrique Donoso, la mujer chilena tiene actualmente mayores posibilidades de desarrollo educacional, laboral, académico y profesional que, sumado al fácil acceso para el control de su fertilidad, hace que la tasa global de fecundidad, por ejemplo, en 2004, fuese de 1,9 hijos por mujer. Lo preocupante es que

\footnotetext{
74 Pieper, 2009, p. 91.

75 Rojas - JiLes, 1992, p. 127.

76 Connelly, 2008b, p. 170.
} 
esta cifra es inferior a la tasa de recambio poblacional que es de 2,1 hijos por mujer, lo que significa que los hijos nacidos por cada mujer no alcanzarían para renovar la población al momento del fallecimiento de sus progenitores. Todo lo anterior sugiere que las políticas gubernamentales en el campo social, salud, vivienda, educación, etc., deben ahora ser reformuladas y orientadas a estimular la fecundidad y a fortalecer el concepto de familia ${ }^{77}$.

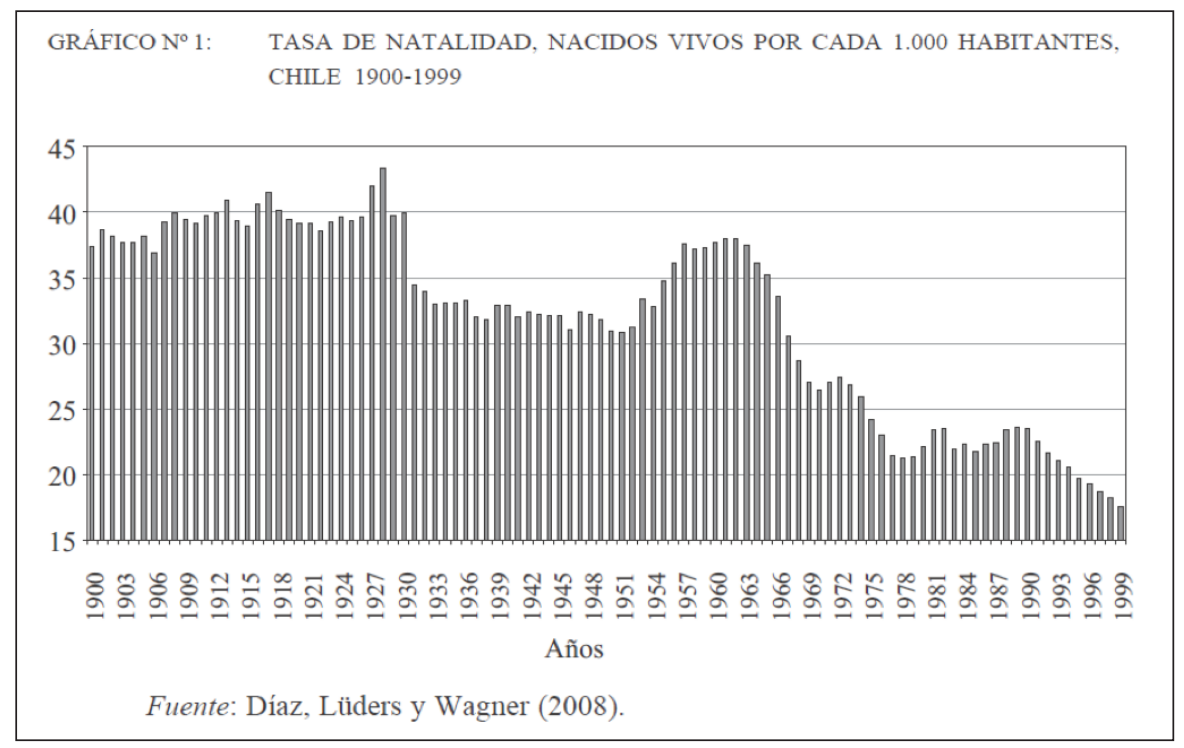

Queda claro que el desarrollo del "primer mundo" aún no ha llegado a Chile, pero ifue el factor natalidad un punto de avance significativo hacia este objetivo o -como lo cuestionaron algunos-, será que, más bien, la consecuencia de esta política es el envejecimiento de la población chilena, lo que debilitaría, a la larga, los elementos humanos para comenzar o continuar con el desarrollo? Sin duda alguna, preguntas abiertas como las anteriores nos invitan a profundizar en las articulaciones propias de la Guerra Fría latinoamericana, de su dimensión cultural y, por ende, de los actores involucrados que recibieron y se apropiaron de un modelo de transformación social que pensaban haría de Chile un país de primer orden.

Historiadores como Matthew Connelly afirman que en el periodo de la Guerra Fría, en cierta manera, se utilizaron este tipo de modelos de desarrollo internacionales para articular nuevas visiones de las relaciones Norte-Sur. Estos aspectos -insiste Connelly- sirven a la historiografía para investigar cómo se utilizó la política de población en el período y, desde allí, las tensiones que se dieron entre múltiples aspectos. Por ejemplo, dentro y entre el feminismo, la eugenesia, el ecologismo y el disque "desarrollo". Conocer la interacción de -éstos en los espacios globales y locales es un tema que los estudiosos apenas han comenzado a explorar ${ }^{78}$.

\footnotetext{
77 Donoso, 2007, pp. 72-75.

78 Connelly, 2003, p. 123.
} 


\section{REFERENCIAS BIBLIOGRÁFICAS}

Ahumada, Jorge

1958 En vez de la Miseria. Editorial del Pacífico. Santiago de Chile.

Arne Westad, Odd

2005 The Global Cold War: Third World Interventions and the Making of Our Times. Cambridge. Cambridge University Press.

2010 The Cambridge History of the Cold War, vol. I. Cambridge. Cambridge University Press.

Avendaño, Onofre

1975 Desarrollo histórico de la planificación de la familia en Chile y en el mundo. APROFA. Informe Mecanografiado. Santiago.

Bojorge, Horacio

1969 "Un segundo Vietnam: Ante la guerra demográfica". El Ciervo. Barcelona, año $18, n^{\circ} 183$. pp. 6-7.

CABEzas, Betty

1967 América Latina: una y múltiple. Tomo I. Santiago de Chile. DESAL Herder.

Castro Arcos, Javier - Ramos Rodríguez, Froilán

2014 “La Alianza para el Progreso en Chile y Venezuela, 1961-1963”. Tiempo y Espacio. Caracas, $n^{\circ}$ 62, julio-diciembre, pp. 93-138.

CALANDRA, Benedetta

2011 'La Ford Foundation y la 'guerra fría cultural' en América Latina (19591973)". Americanía: Revista de Estudios Latinoamericanos. Sevilla, nº 1, pp. 8- 25.

CAlandra, Benedetta - Marina, Franco (eds.)

2012 La Guerra Fría cultural en América Latina. Desafios y límites para una nueva mirada de las relaciones interamericanas. Buenos Aires. Editorial Biblos.

CobBs-Hoffman, Elizabeth

1998 All You Need is Love: The Peace Corps and the Spirit of the 1960s. Cambridge. Harvard University Press.

ConNelly, Matthew

2003 "Population Control Is History: New Perspectives on the International Campaign to Limit Population Growth". Comparative Studies in Society and History. Cambridge, vol. 45, n⿳0 1, pp. 122-147.

2008a "Controlling Passions". The Wilson Quarterly. Washington, vol. 32, n 3 , pp. 60-66.

2008b Fatal Misconception: The Struggle to Control World Population. Cambridge. Harvard University Press.

Consuegra, Josué

1969 El control de natalidad como arma del imperialismo. Bogotá. Editorial Galena. 
Correa, Sofía (ed.)

2001 Historia del siglo XX chileno: Balance paradojal. Santiago de Chile. Editorial Sudamericana.

Cueto, Marcos (ed.)

1994 Missionaries of Science: The Rockefeller Foundation and Latin America. Philanthropic Studies. Bloomington. Indiana University Press.

Cull, Nicholas J.

2008 The Cold War and the United States Information Agency. American Propaganda and Public Diplomacy, 1945-1989. Cambridge. Cambridge University Press.

DíAz, J. - LÜDERs, R. - Wagner, G.

2008 La República en Cifras. Santiago de Chile. Mimeo.

Donoso, Enrique

2007 "Descenso de la natalidad en Chile: Un problema país". Revista chilena de Obstetricia y Ginecología. Santiago de Chile, vol. 72, n² 2, pp. 73-75.

FeLITTI, Karina

2008 "La 'explosión demográfica' y la planificación familiar a debate: Instituciones, discusiones y propuestas del centro y la periferia". Revista Escuela de Historia. Salta, vol.7, $\mathrm{n}^{\circ} 2$, pp. 1-20.

FERMANDOIS, Joaquín

1998 “¿Peón o actor? Chile en la Guerra fría (1962-1973)”. Estudios Públicos. Santiago, $\mathrm{n}^{\circ}$ 72, pp. 149-171.

Gavin J., Francis - Lawrence A., Mark (eds.)

2014 Beyond the Cold War: Lyndon Johnson and the New Global Challenges of the 1960s. New York. Oxford University Press.

Garrard-Burnet, Virginia - Lawrence, Mark A. - Moreno, Julio (eds.)

2013 Beyond the Eagle's Shadow: New Histories of Latin America's Cold War. Albuquerque. University of New Mexico Press.

GILBERT, Joseph - Spencer, Daniela (eds.)

2008 In from the Cold: Latin America's New Encounter with the Cold War. Durham. Duke University Press.

GUTIÉRREZ, Roberto

2004 "Walt W. Rostow: Réquiem por un historiador económico." Ciencia Ergo Sum. México D.F., vol. 10, no 3, pp. 295-303.

GóngORA, Mario

1981 Ensayo histórico sobre la noción de Estado en Chile en los siglos XIX y XX. Santiago de Chile. Editorial La Ciudad.

Harmer, Tanya - Riquelme, Alfredo (eds.).

2014 Chile y la Guerra Fría global. Santiago de Chile. RIL Editores.

HART, Justin

2012 Empire of Ideas: The Origins of Public Diplomacy and the Transformation of U.S. Foreign Policy. Oxford-New York. Oxford University Press. 
KASUN, Jacqueline

1988 The war against population: The economics and ideology of population control. San Francisco. Ignatius Press.

KORRY, Edward

1998 "Los Estados Unidos en Chile y Chile en los Estados Unidos. Una retrospectiva política y económica (1963-1975)”. Estudios Públicos. Santiago de Chile, ${ }^{\circ} 72$, pp. 17-74.

Lagos, Claudia

2001 El aborto en Chile, el deber de parir. Santiago de Chile. LOM Ediciones.

LANGLAND, Victoria

2013 Speaking of Flowers: Student Movements and the Making and Remembering of 1968 in Military Brazil. Durham. Duke University Press

LARRAÍn, Hernán

1966 “Campaña birthcontrolista del SNS?”. Mensaje. Santiago de Chile, vol. XV, $\mathrm{n}^{\circ} 146$, pp. 44-46.

LATHAM, Michael E.

2000 Modernization as Ideology. American Social Science and "Nation Building" in the Kennedy Era. Chapel Hill, NC. The University of North Carolina Press.

2010 The Cambridge History of the Cold War, vol. 2. Cambridge. Cambridge University Press.

2011 The Right Kind of Revolution: Modernization, Development, and U.S. Foreign Policy from the Cold War to the Present. New York. Cornell University Press.

LEVInson, Jerome - De Onis, Juan

1972 La Alianza Extraviada. México. Fondo de Cultura Económica.

LEwIS GADDIS, John

2012 Nueva Historia de la Guerra Fría. México. Fondo de Cultura Económica.

LOWENTHAL, Abraham

2010 "Estados Unidos de América Latina, 1960-2010: De la pretensión hegemónica a las relaciones diversas y complejas". Foro Internacional. México, vol. L, $\mathrm{n}^{\mathrm{o}} 3-4$, pp. 552-626.

Mattelart, Armand

1965 El reto espiritual de la explosión demográfica. Santiago de Chile. Editorial del Pacífico.

Molina, Sergio

1972 El Proceso de Cambio en Chile. Santiago de Chile. Instituto Latinoamericano de Planificación Económica y Social - Editorial Universitaria.

MuÑoz, Luis Joaquín

1977 “El paradigma americano de la modernización". Revista Española de la Opinión Pública. Madrid, nº 47, pp. 77-90.

MYRDAL, Gunnar

1974 “What is Development?". Journal of Economic Issues. Arkansas, Association for Evolutionary Economic, vol. 8, nº 4, pp. 729-736. 
NeCochea LóPEZ, Raúl

2010 "Anticonceptivos en el Sur, Seguridad Nacional en el Norte". Salud colectiva. Lanús, vol. 6, $\mathrm{n}^{\mathrm{o}}$ 1, enero/abril, pp.103-111.

2014 A History of Family Planning in Twentieth-Century Peru. North Carolina. The University of North Carolina Press.

Niño, Antonio

2009 "Uso y abuso de las relaciones culturales en la política internacional". Ayer. Madrid, $n^{\circ} 75 / 3$, pp. 25-61.

PIEPER, Jadwiga

2009 The politics of motherhood: Maternity and women's rights in Twentieth-century Chile. Pittsburgh. University of Pittsburgh Press.

PinTo, Aníbal

1968 Política y Desarrollo. Santiago de Chile. Editorial Universitaria.

Poblete Troncoso, Moisés

1967 La Explosión demográfica en América Latina. Buenos Aires. Editorial Schapire.

Prebisch, Raúl

1992 "Conferencia Regional Latinoamericana de Población. Intervención del Dr. Raúl Prebisch. Notas de población". Revista Latinoamericana de Demografia. CELADE. Santiago de Chile, pp. 2-25.

PuRCELl, Fernando

2014a "Connecting Realities: Peace Corps Volunteers in South America and the Global War on Poverty during the 1960s". Historia Crítica. Bogotá, nº 53, pp. 129-154. 2014b "Guerra Fría, motivaciones y espacios de interacción. El caso del Cuerpo de Paz de Estados Unidos en Chile, 1961-1970". En Hamer - Riquelme (eds.), Chile y la Guerra Fría global. Santiago de Chile. RIL Editores.

Quinche, Carlos

2009 "La modernización en las relaciones EE.UU. América Latina durante la administración Kennedy: una mirada a la luz de Norbert Elias". Revista Colombiana de Sociología. Bogotá, vol. 32, nº 1, pp. 95-113.

Quintana, María Soledad

2010 Sexo seguro, cuerpos disciplinados. Quito. Editorial FLACSO Ecuador.

Paulo VI, Papa

1968 Humanae Vitae: Carta Encíclica de su Santidad el Papa Pablo VI sobre la regulación de la natalidad. Madrid. Editorial Mundo Cristiano.

REY TRISTÁN, Eduardo

2012 Estados Unidos y América Latina durante la Guerra Fría: la dimensión cultural. En Calandra - Franco (eds.), La Guerra Fría cultural en América Latina. Desafíos y límites para una nueva mirada de las relaciones interamericanas. Buenos Aires. Editorial Biblos.

RoJas, Claudia

2009 "Lo global y lo local en los inicios de la planificación familiar en Chile". Estudios Avanzados. Santiago de Chile, nº II, pp. 7-27. 
RoJAs, Claudia - JiLes, Ximena

1992 De la Miel a los Implantes: Historia de las políticas de regulación de la fertilidad en Chile. Santiago de Chile. CORSAPS.

RoJAs, Diana Marcela

2010 "La Alianza para el Progreso en Colombia". Análisis político. Bogotá, n 70, pp. 91-124.

ROMERo Hernán

1969 "El crecimiento de la población y el control de la natalidad". Cuadernos Médico-Sociales. Santiago de Chile, vol. X, nº 1, pp. 5-21.

SAUNDERS, Frances Stonor

2001 La CIA y la Guerra Fría cultural. Barcelona. Ed. Debate.

SAuvy, Alfred

1963 Malthus et les deux Marx. Paris. Denoël.

STYcos, Mayone

1968 "Opposition to Family Planning in Latin America: Conservative Nationalism”. Demography. New York, vol. 5, nº 2, pp. 846-854.

TINSMAN, Heidi

2009 La tierra para el que la trabaja: género, sexualidad y movimientos campesinos en la Reforma agraria chilena. Santiago de Chile, LOM Ediciones.

Tomic, Radomiro

1966 "El control de la Natalidad". Política y Espíritu. Santiago de Chile, año XX, n 295 (agosto-septiembre), pp. 16-24.

VAZ DA Costa, Rubens

1970 "Crecimiento de la población y desarrollo económico". Demografía y economía. México D.F., vol. 4, nº 2, pp. 210-226.

VIAL, Gonzalo

1981 Historia Contemporánea de Chile (1891-1973), vol. I, tomo II. Santiago de Chile. Editorial Santillana.

VIEL, Benjamín

1966 La explosión demográfica: ¿Cuántos son demasiados? Santiago de Chile. Editorial Universitaria. 